\title{
Observing UT1-UTC with VGOS
}

\author{
Rüdiger Haas ${ }^{1 *} \mathbb{B}$, Eskil Varenius ${ }^{1}$, Saho Matsumoto ${ }^{2}$ and Matthias Schartner ${ }^{3,4}$
}

\begin{abstract}
We present first results for the determination of UT1-UTC using the VLBI Global Observing System (VGOS). During December 2019 through February 2020, a series of $1 \mathrm{~h}$ long observing sessions were performed using the VGOS stations at Ishioka in Japan and the Onsala twin telescopes in Sweden. These VGOS-B sessions were observed simultaneously to standard legacy S/X-band Intensive sessions. The VGOS-B data were correlated, post-correlation processed, and analysed at the Onsala Space Observatory. The derived UT1-UTC results were compared to corresponding results from standard legacy S/X-band Intensive sessions (INT1/INT2), as well as to the final values of the International Earth Rotation and Reference Frame Service (IERS), provided in IERS Bulletin B. The VGOS-B series achieves 3-4 times lower formal uncertainties for the UT1-UTC results than standard legacy S/X-band INT series. The RMS agreement w.r.t. to IERS Bulletin B is slightly better for the VGOS-B results than for the simultaneously observed legacy S/X-band INT1 results, and the VGOS-B results have a small bias only with the smallest remaining standard deviation.
\end{abstract}

Keywords: UT1-UTC, VLBI intensives, VLBI Global Observing System (VGOS), VGOS twin telescopes

\section{Introduction}

Geodetic Very Long Baseline Interferometry (VLBI) (Sovers et al. 1998) is to date the only space geodetic technique that can observe all earth orientation parameters (EOP). This is primarily due to that VLBI directly observes natural radio sources in the International Celestial Reference Frame (ICRF, Charlot et al. 2020) with radio telescopes on the surface of the earth, i.e. in the terrestrial reference frame (ITRF, Altamimi et al. 2016). The EOP are the transformation parameters between these two frames. The daily rotation of the earth is the EOP that is most difficult to predict due to rapidly varying geophysical excitation. It is usually reported as difference between UT1 and Universal Time Coordinated (UTC), and a precise and accurate monitoring of this parameter is of great importance for satellite navigation systems and satellite orbit determination (Bradley et al. 2016).

The International VLBI Service for Geodesy and Astrometry (IVS) (Nothnagel et al. 2017) therefore organises dedicated regular observing sessions to determine

\footnotetext{
*Correspondence: rudiger.haas@chalmers.se

${ }^{1}$ Department of Space, Earth and Environment, Chalmers University of Technology, Onsala Space Observatory, SE-439 92 Onsala, Sweden Full list of author information is available at the end of the article
}

UT1-UTC, so that the International Earth Rotation and Reference Frames Service (IERS 2021) can produce precise, accurate and reliable data series of UT1-UTC with low latency that scientific users and society at large can use. These IVS session are the so-called IVS Intensive sessions (INT), which have been observed routinely since decades with the legacy S/X-band VLBI system that the IVS organises. The legacy S/X-band VLBI system works with single-polarisation dual-frequency observations in the $2 \mathrm{GHz}$ (S-band) and $8 \mathrm{GHz}$ (X-band) frequency range. The INT series make use of long east-west oriented baselines due to their high sensitivity for UT1. The two main series are INT1, usually observed on weekdays on the baseline between Kokee (Hawaii, US) and Wettzell (Germany), and INT2, usually observed on weekends on the baseline between Wettzell (Germany) and Ishioka (Japan). Before Ishioka was involved in INT2, instead the station Tsukuba (Japan) was part of this series. There is also a third INT series, INT3, which observes on Monday mornings usually with a three-station network. During the years, a number of variations were seen in terms of which stations were involved, mainly due to replacing stations for times of station outages due to e.g. maintenance.

While the standard INT are observed with the IVS legacy S/X-band system, during the last years new stations 
equipped with the next generation VLBI system called the VLBI Global Observing System (VGOS) have been constructed. The overall goals of VGOS are to contribute to the Global Geodetic Observing System (GGOS, Plag and Pearlman 2009) by providing $1-\mathrm{mm}$ position accuracy on a global scale and continuous measurements for time series of station positions and EOP (Niell et al. 2006). The most challenging aspects and restricting factors for geodetic VLBI today are atmospheric turbulence (Nilsson and Haas 2010) and radio source structure (Anderson and Xu 2018). To better address these, the VGOS system has been developed (Niell et al. 2006; Petrachenko et al. 2009; Niell et al. 2018). VGOS makes use of very fast-slewing telescopes $(12 \%$ s in azimuth and $6 \%$ in elevation) with broadband receiving systems covering four frequency ranges, and dual polarization $(\mathrm{H} / \mathrm{V})$ capability (Petrachenko et al. 2009; Niell et al. 2018).

Ishioka (Wakasugi et al. 2019) is one of these stations. Furthermore, it can exchange the receiving system, i.e. Ishioka can be used for some months of the year as legacy S/X-band station and for other months of the year as VGOS station. Another example of VGOS stations are the Onsala twin telescopes (OTT, Haas et al. 2019), which are the currently only operational VGOS twin telescopes. The goals of VGOS twin telescopes are manifold. Twin telescopes can achieve continuous 24/7 observations, even when one of the telescopes may need maintenance. They can work in parallel and thus achieve a high degree of robustness for the VGOS products. And they can observe in parallel together with different networks of partner telescopes, thus observing in different directions and therefore sense the local atmosphere and atmospheric turbulence spatially and temporally better than one single telescope alone. Both Ishioka and the OTT are participating routinely in the IVS VGOS operations series (VO). In discussions with the IVS coordinating center in late 2019, the idea came up to start test observations to make use of VGOS for Intensive sessions and thus explicitly the determination of UT1-UTC. Using the Onsala twin telescopes in this so-called VGOSB series allows simultaneous determination of UT1-UTC with two parallel long east-west baselines connecting to Ishioka.

\section{Scheduling and observing the VGOS-B sessions}

For the period December 2019 through February 2020 in total twelve VGOS-B sessions were scheduled. The scheduling was done with the software VieSched++ (Schartner and Böhm 2019) involving the VGOS stations ISHIOKA (Is) in Japan and ONSA13NE (Oe) and ONSA13SW (Ow) in Sweden. The schedules were prepared to be simultaneous to standard INT1 observations. During scheduling, special emphasis was given to include observations at the corners of the mutually visible sky since these observations are known to provide the most impact on the precision of the derived UT1-UTC results (Uunila et al. 2012; Gipson and Baver 2015). As an example, Fig. 1 depicts the observations of VGOS-B session B20023 in a baseline mid-point representation (Nothnagel and Campbell 1991; Leek et al. 2015). To achieve such a schedule, a special scheduling algorithm was used. The schedule starts by observing a source at one of the two corners of the mutually visible sky (see scan \#1 in Fig. 1), followed by scans selected using standard geodetic scheduling optimization. Every ten minutes, the algorithm forces a scan of a source located in one of the corners in case a reasonably bright source is visible and the area around the corner was not observed recently, see Fig. 1 scan \#8, \#13, \#15, \#20, etc. Therefore, it is ensured that observations at the corners of the mutually visible sky are well represented in the schedule.

Due to the modern and fast-slewing VGOS telescopes, the number of scheduled observations per baseline was more than 50 during the $1 \mathrm{~h}$ long sessions. This is significantly more than the usually $20-25$ observations during $1 \mathrm{~h}$ long INT1 and INT2 sessions with legacy S/X-band stations. We note that the VGOS-B sessions analysed in this paper were scheduled assuming standard VO-session recording which limits the number of scans per unit time. Future VGOS-B sessions will be further optimised

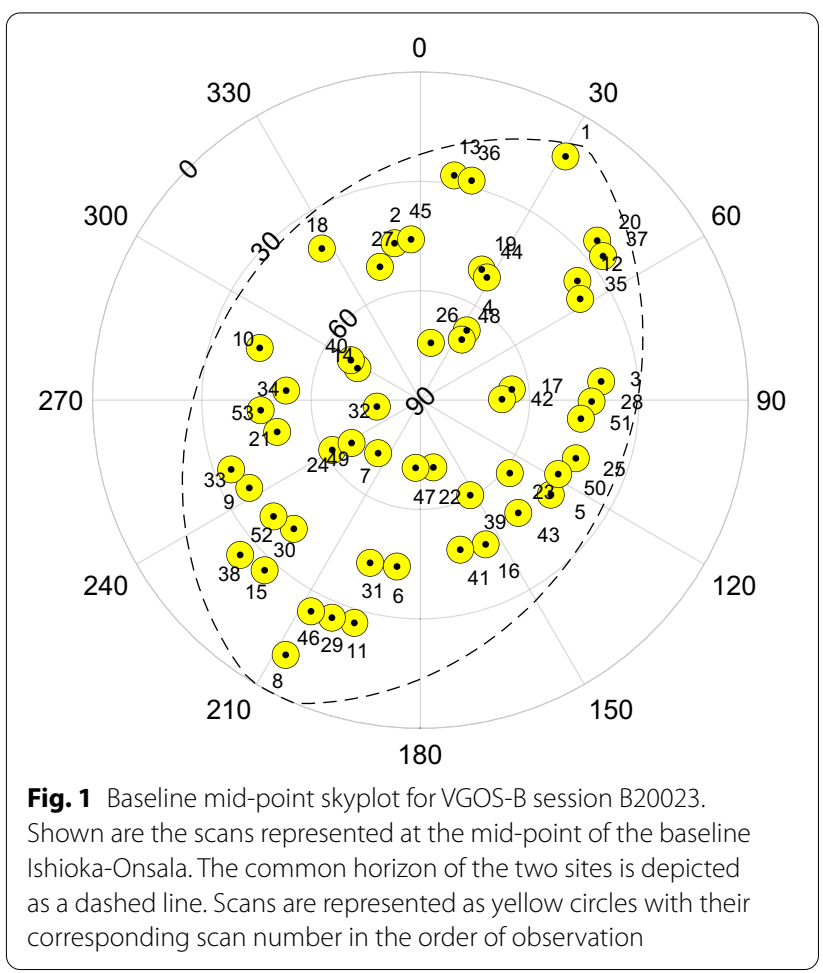


for the Is-Oe/Ow systems and can therefore schedule $\sim 100$ observations per baseline per hour, fully utilising the fast slewing speed.

Table 1 lists these twelve VGOS-B sessions and their INT1 counterparts, including the stations involved. From simulations with VieSched ++ the formal uncertainties of the UT1-UTC estimates are 4-5 $\mu$ s for the VGOS-B sessions, while they are a factor of 2-3 worse for the legacy $\mathrm{S} / \mathrm{X}$-band intensive schedules. Unfortunately, during four of the twelve VGOS-B sessions technical problems occurred at Ishioka, so that the observed raw data were not complete, see the comments in the table.

\section{Correlation and post-correlation analysis}

The observed raw data of the Ishioka station were transferred electronically to the VLBI correlator at the Onsala Space Observatory. The software correlator DiFX version 2.6.1 by Deller et al. (2011) was used for correlation and the Haystack Observatory Postprocessing System (HOPS, MIT/Haystack 2020) version 3.21 was used for the post-correlation analysis. The data were processed according to the VGOS Data Processing Manual (MIT/Haystack 2019). This included forming the pseudo-stokes I polarisation product, from the recorded $\mathrm{H}$ and $\mathrm{V}$ data, to account for parallactic-angle differences. Since Ishioka had only observed VGOS band-A during B20007, see Table 1, this session could not be processed. The last three experiments had reduced or no signal V-pol for Is, see Table 1 . Therefore, the determination of the small additional sampler phase-offsets was not possible with the standard

Table 1 VGOS-B sessions in December 2019 through February 2020, and their simultaneously observed INT1 counterparts

\begin{tabular}{lllll}
\hline Code & Stations & Comment & Code & Stations \\
\hline B19344 & Is-Oe-Ow & 119344 & $\mathrm{Mk}-\mathrm{Wz}$ \\
B19351 & Is-Oe-Ow & 119351 & $\mathrm{Kk}-\mathrm{Wz}$ \\
B19357 & Is-Oe-Ow & 119357 & $\mathrm{Kk}-\mathrm{Wz}$ \\
B19364 & Is-Oe-Ow & & 119364 & $\mathrm{Kk}-\mathrm{Wz}$ \\
B20007 & Is-Oe-Ow & (Is-data: only VGOS band-A) & 120007 & $\mathrm{Kk}-\mathrm{Wz}$ \\
B20013 & Is-Oe-Ow & & 120013 & $\mathrm{Kk}-\mathrm{Wz}$ \\
B20023 & Is-Oe-Ow & 120023 & $\mathrm{Kk}-\mathrm{Wz}-\mathrm{Sv}$ \\
B20027 & Is-Oe-Ow & & 120027 & $\mathrm{Kk}-\mathrm{Wz}$ \\
B20037 & Is-Oe-Ow & & 120037 & $\mathrm{Kk}-\mathrm{Wz}-\mathrm{Sv}$ \\
B20044 & Is-Oe-Ow & (Is-data: only H-polarization) & 120044 & $\mathrm{Kk}-\mathrm{Wz}$ \\
B20048 & Is-Oe-Ow & (Is-data: only H-polarization) & 120048 & $\mathrm{Mk}-\mathrm{Wz}$ \\
B20055 & Is-Oe-Ow & (Is-data: only H-polarization) & 120055 & $\mathrm{Kk}-\mathrm{Wz}$
\end{tabular}

The stations are ISHIOKA (Is), ONSA13NE (Oe), ONSA13SW (OW), KOKEE (Kk), WETTZELL (Wz), SVETLOE (SV) and MK-VLBA (Mk). The VGOS-B baseline with the longest extension in the XY-plane, and thus most sensitive for the determination of UT1-UTC, is Is-Ow with about $7773 \mathrm{~km}$. The INT1-baselines with the longest extension in the XY-plane are Mk-Wz with about 10,136 km and Kk-Wz with about $10,072 \mathrm{~km}$ procedures employed for VGOS experiments. However, since these phase-offsets are small and usually very stable over a time scale of several months, we used the phase-offset values for B20037 and applied these also to the latter experiments. The resulting delays are, as expected, weaker than for the preceding sessions (due the lack of V-pol signal, and possibly sub-optimal phase-offsets), but as the results appear reasonable, we still could include them in the subsequent data analysis. The short $(75 \mathrm{~m})$ baseline Oe-Ow suffered from disturbing local radio frequency interference (RFI). The full VGOS band-A, and a few channels in other VGOS-bands, were therefore excluded from Oe-Ow post-processing, thus deteriorating the delay accuracy on this baseline. Finally, the VLBI delay observations were exported to vgosDb format (Bolotin et al. 2015). The corresponding tools of the $\nu$ Solve software package version 0.7.1 (Bolotin et al. 2012) were used to calculate and add the theoretical delays (vgosDbCalc) as well as the supplemental data from the observing station logfiles (vgosDbProcLogs). We note that while Oe and Ow are equipped with Cable Delay Measurement Systems (CDMS) which stores delay corrections in the observing logfiles, Is currently does not measure such corrections. Therefore, proxy-cable corrections were derived using the Is phase-calibration data as described in VGOS Data Processing Manual (MIT/Haystack 2019).

\section{Data analysis and results}

The geodetic data analysis was performed with the ASCoT software (Artz et al. 2016). We analysed both the VGOS-B sessions, as well as all INT1 and INT2 sessions in the time range December 2019 through February 2020. Eleven out of the twelve VGOS-B sessions could be analysed, but not B20007 where Ishioka had only observed VGOS band-A and no vgosDb could be created.

A standard analysis approach for INT sessions was used:

- All station coordinates were kept fixed on their a priori values, i.e. the IVS VTRF2020b (BKG 2020) values. For the OTT we used the corresponding VTRF2020b coordinates that were determined through dedicated short-baseline interferometry campaigns (Varenius and Haas 2020).

- The radio source positions were kept fixed on their ICRF3 (Charlot et al. 2020) values.

- A priori information for the EOP was taken from the most recent update of the EOP 14 C04 series (Bizouard et al. 2019). 
- We fixed the clock for one of the stations as reference, while estimating 2nd order clock polynomials for the other stations involved.

- We estimated one zenith wet delay parameter for each of the stations but atmospheric gradients were not estimated.

- The UT1-UTC parameter was estimated.

First, we investigated the agreement of the results of the two parallel baselines Is-Oe and Is-Ow by analyzing the two baselines individually and together. The short baseline Oe-Ow was excluded from any analysis due to the previously mentioned RFI problems. Figure 2 depicts the corrections to the a priori UT1-UTC from these three approaches. The results of all the analysis of the two individual baselines, i.e. Is-Oe and Is-Ow, agree within their formal errors. The analysis of both baselines together provides an average of the two result of the two individual baselines. With $4 \mu \mathrm{s}$ it also gives the lowest median formal uncertainty of UT1-UTC, while the median formal uncertainties for the individual baselines are $6 \mu$ s for Is-Oe and Is-Ow. These formal uncertainties correspond well with the results from simulations performed with VieSched ++ . We also tested an additional analysis strategy where we estimated common ZWD parameters for the twin stations, but this did not give significantly different results. In the following we used the results of analysing both baselines together.

Besides performing our own data analysis of the INT1 and INT2 sessions, we also downloaded the results of the corresponding analyses that were provided by five of the IVS Analysis Centres (IVS ACs). These were the

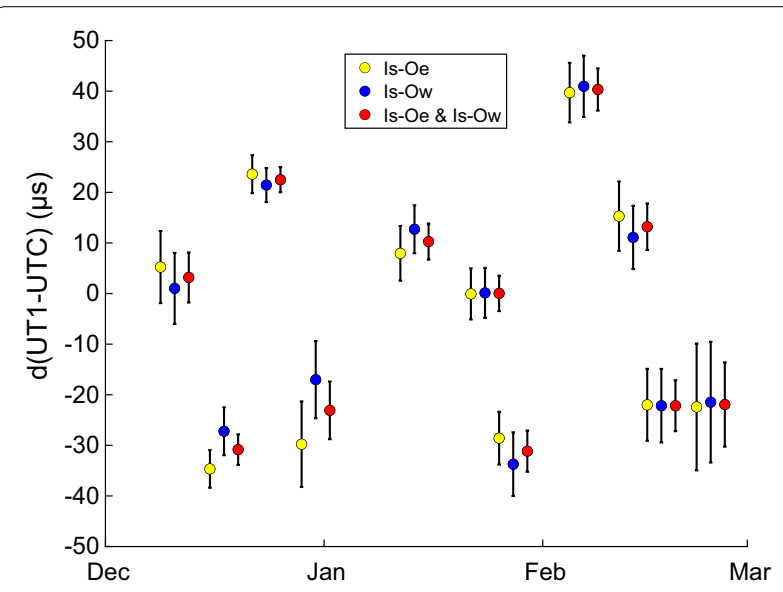

Fig. 2 Comparison of UT1-UTC corrections to the a priori values derived from analysing the VGOS-B baselines individually, i.e. Is-Oe (yellow) and Is-Ow (blue), and in a common analysis Is-Oe \& Is-OW (red). The symbols are offset along the $x$-axis to improve readability of the graph. The uncertainties shown are $1 \sigma$
Bundesamt für Kartographie und Geodäsie (BKG), the NASA Goddard Space Flight Center (GSF), the United States Naval Observatory (USN), the Geospatial Information Authority of Japan (GSI), and the Institute of Applied Astronomy of the Russian Academy of Sciences (IAA). These IVS ACs routinely analyze the INT sessions and provide their results to the IERS for the determination of IERS rapid and final products (Luzum and Gambis 2014), IERS Bulletin A and IERS Bulletin B, respectively. All except GSI provide results for INT1 and INT2 sessions. The (few) results with formal uncertainties larger than $100 \mu$ s were excluded from further comparisons.

The left plot in Fig. 3 presents the UT1-UTC times series. The IERS Bulletin $\mathrm{B}$ values are shown as grey stars and the VGOS-B results as red circles. The INT1/ INT2 results of the five IVS ACs are depicted in light blue as squares (BKG), upward-pointing triangles (GSF), downward-pointing triangles (USN), left-pointing triangles (GSI), and right-pointing triangles (IAA). Our own analysis results of the INT1/INT2 data are presented as light red diamonds. To increase the readability of this graph, all time series, except the VGOS-B one, are offset by $-3 \mathrm{~ms}$ each from the series shown directly above. The formal uncertainties of the presented results are on the order of a few $\mu$ s to several tens of $\mu$ s and thus not visible in this scale since UT1-UTC is changing a lot during the presented time interval. However, from a first glance at the graph it is obvious that all presented results show a similar signature and agree rather well. The VGOS-B results (red circles) do not show any obvious deviation with respect to the other data sets.

More insight is gained when investigating the differences between the individual UT1-UTC results and the IERS Bulletin B values. The latter are provided as daily values at epoch 00:00 UTC, while the INT1/INT2 and VGOS-B results are given at the respective observation times of the corresponding session. Thus, to evaluate the agreement between the individual UT1-UTC series and IERS Bulletin B, the latter had to be interpolated to the epochs of the UT1-UTC series. For simplicity, and since all of the series do not include any high-frequent variations of UT1-UTC, we used a cubic spline interpolation. The right plot in Fig. 3 depicts the times series of the resulting differences explicitly for the simultaneously observed INT1 and VGOS-B sessions.

It becomes clear that all series agree reasonably well with the IERS Bulletin $B$ values, with maximum deviations well within the $\pm 80 \mu$ s interval. Table 2 provides both the mean and median formal uncertainties of the individual series, their root mean square (RMS) agreement w.r.t. the IERS Bulletin B series, the biases, and the remaining scatter after removing the biases, expressed as standard deviation (STD). These statistics are provided 

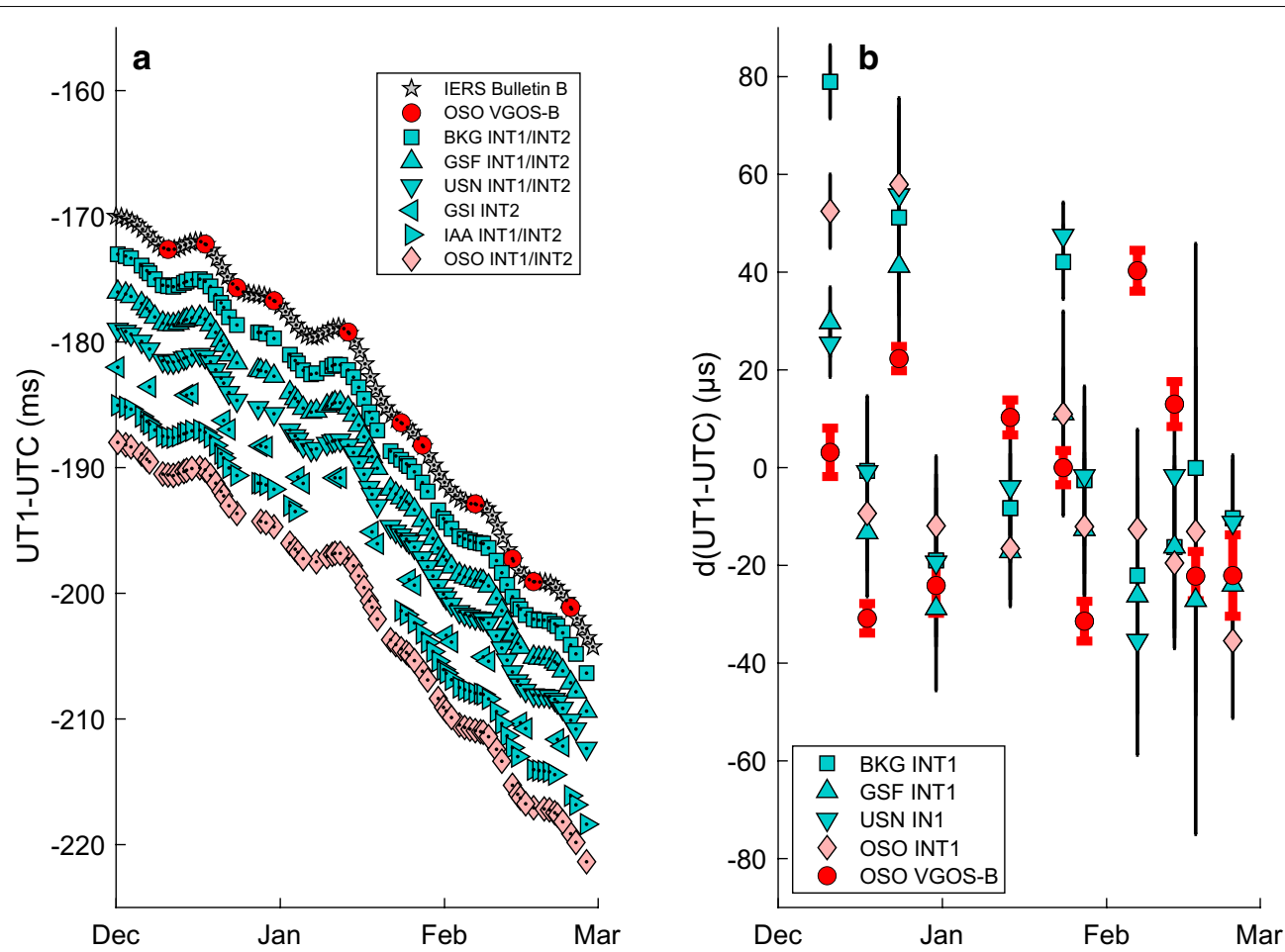

Fig. 3 Left plot a Time series of UT1-UTC for December 2019 through February 2020. Shown are IERS Bulletin B values (grey stars) and the VGOS-B results as (red circles). The light blue symbols show the INT1/INT2 results of BKG (squares), GSF (upward-pointing triangles), USN (downward-pointing triangles), GSI (left-pointing triangles), and IAA (right-pointing triangles). The INT1/INT2 results from OSO are shown as light-red diamonds. To increase readability, all series, except the VGOS-B one, are offset by $-3 \mathrm{~ms}$ each from the series shown directly above. Right plot b Differences with respect to IERS Bulletin B values for simultaneously observed INT1 and VGOS-B sessions

Table 2 Statistics of the UT1-UTC times series. Shown are the mean formal uncertainties $\left(\sigma_{\text {mean }}\right)$, median formal uncertainties $\left(\sigma_{\text {median }}\right)$, the (unweighted) root-mean square agreement (RMS) w.r.t. the IERS Bulletin B series, the mean bias and the standard deviation (STD) after removing the bias

\begin{tabular}{lrrrll}
\hline Series & $\sigma_{\text {mean }}$ & $\sigma_{\text {median }}$ & RMS & Bias & STD \\
\hline All BKG INT1/INT2 & 14.8 & 14.2 & 32.9 & $14.3 \pm 3.4$ & 29.6 \\
All GSF INT1/INT2 & 15.1 & 13.2 & 28.3 & $-2.0 \pm 3.2$ & 28.2 \\
All USN INT1/INT2 & 14.6 & 12.9 & 28.4 & $8.5 \pm 3.1$ & 27.1 \\
All GIS INT2 & 13.5 & 9.2 & 33.6 & $10.2 \pm 6.7$ & 32.0 \\
All IAA INT1/INT2 & 15.0 & 12.2 & 27.8 & $5.0 \pm 3.6$ & 27.3 \\
All OSO INT1/INT2 & 16.5 & 15.4 & 31.0 & $4.5 \pm 3.6$ & 30.6 \\
OSO VGOS-B & 4.5 & 4.2 & 23.2 & $-3.8 \pm 7.2$ & 22.9 \\
Simultaneous OSO INT1 & 16.0 & 14.2 & 28.4 & $-0.8 \pm 9.0$ & 28.3 \\
Simultaneous BKG INT1 & 17.3 & 16.1 & 32.9 & $8.4 \pm 10.1$ & 31.8 \\
Simultaneous GSF INT1 & 18.8 & 16.8 & 24.2 & $-7.6 \pm 7.3$ & 23.0 \\
Simultaneous USN INT1 & 14.3 & 14.4 & 27.9 & $5.4 \pm 9.1$ & 27.4 \\
\hline Allvalues are given in unit & & Th & & &
\end{tabular}

All values are given in unit $\mu \mathrm{s}$. The upper part of the table presents the statistics of all 3 months of INT1/INT2, i.e. December 2019 through February 2020, while the lower part focuses specifically on the eleven simultaneously observed INT1 sessions in the upper part of the table for all INT1/INT2 from December 2019 through February 2020, and in the lower part of the table specifically focusing on the eleven simultaneously observed INT1 sessions. The VGOS-B data show both the smallest uncertainties, a small bias, as well as the best agreement in terms of RMS and STD.

\section{Conclusions and outlook}

We scheduled, observed, correlated, post-correlation processed and analysed a number of VGOS sessions for the determination of UT1-UTC. These are the first sessions involving VGOS twin telescopes for this purpose. The involved VGOS stations were Ishioka (Is) in Japan and the Onsala twin telescopes (Oe, Ow) in Sweden, forming two parallel long east-west oriented baselines, i.e. Is-Oe and Is-Ow. We compared the derived UT1UTC values from these VGOS-B sessions with corresponding results from our own analysis of INT1/INT2 data, the corresponding INT1/INT2 results of five other IVS ACs, and the final values of the IERS Bulletin B. We investigated both the results of the simultaneous VGOS-B and INT1 sessions, as well with 3 months of INT1/INT2 sessions. The mean and median formal 
uncertainties of the VGOS-B derived UT1-UTC results are three to four times lower than the corresponding formal uncertainties of the INT1/INT2 results. This is in good agreement with simulations performed based on the actual schedules. The smallest formal uncertainties are achieved when analysing the two parallel baselines Is-Oe and Is-Ow together. The RMS agreement w.r.t. to IERS Bulletin B is slightly better for the VGOS-B results than for the simultaneously observed legacy S/X-band INT1 results and the VGOS-B results have a small bias only with the smallest remaining standard deviation. Compared to the full 3 months of INT1/INT sessions, the RMS and STD improvements are even on the order of $30-40 \%$. This improvement confirms the simulation study of Corbin and Haas (2019) where various scenarios of VGOS observations of UT1-UTC were investigated. Investigating 6 months of intensive observations by simulations, Corbin and Haas (2019) found an improvement of the UT1-UTC accuracy of more than $40 \%$ when simulating VGOS intensive sessions including one VGOS twin telescope station, compared to standard S/X-band legacy intensives. The good agreement of the VGOS-B results with IERS Bulletin B is remarkable since the VGOS-B baselines are significantly shorter, and thus less sensitive for the determination of UT1-UTC, than the INT1 baselines. Furthermore, the good agreement of the VGOS$B$ results with IERS Bulletin B is also remarkable since the INT1/INT2 results provided by the operational IVS ACs are input data for the determination of the IERS Bulletin B, while the VGOS-B results are not. However, more VGOS-B sessions are necessary to verify this good agreement. Therefore a new series of VGOSB sessions has started in late 2020 and is expected to continue through the spring of 2021. With even more $(\sim 100)$ observations per baseline per hour, we expect these sessions to further improve the determination of UT1-UTC. Finally, we note the possibility of Oe and Ow participating simultaneously in two different UT1UTC sessions, a unique capability of the twin telescopes sites which can hopefully be investigated for upcoming VGOS intensive sessions.

\section{Acknowledgements}

We acknowledge advice given by Mike Titus, Simone Bernhart, John Barret and Sergei Bolotin regarding correlation and post-processing of the VGOS B-sessions. We thank the editor and two reviewers for their constructive feedback that helped to improve the manuscript.

\section{Authors' contributions}

$\mathrm{RH}, \mathrm{EV}, \mathrm{SM}$ and MS organised VGOS-B series, in coordination with the IVS Coordinating Center. MS scheduled the VGOS-B experiments. EV performed the VLBI data correlation and post-correlation analysis and created the final vgos Db. RH analysed the vgosDB with ASCoT and did the comparison analyses. All authors read and approved the final manuscript.

\section{Availability of data and materials}

The vgosDbs analysed in this study are publically available via the IVS webpages. The IERS Bulletin B results are available via the IERS webpages. The individual UT1-UTC series of the IVS ACs are available from the IVS.

\section{Declarations}

\section{Competing interests}

The authors declare that there are no competing interests.

\section{Author details}

${ }^{1}$ Department of Space, Earth and Environment, Chalmers University of Technology, Onsala Space Observatory, SE-439 92 Onsala, Sweden. ${ }^{2}$ Geospatial Information Authority of Japan, 1 Kitasato, Tsukuba 305-0811, Japan. ${ }^{3}$ Department of Geodesy and Geoinformation, TU Wien, Wiedner Hauptstraße 8-10, 1040 Vienna, Austria. ${ }^{4}$ Present Address: Institute of Geodesy and Photogrammetry, ETH Zürich, 8093 Zurich, Switzerland.

Received: 4 December 2020 Accepted: 10 March 2021

Published online: 25 March 2021

\section{References}

Altamimi Z, Rebischung P, Métivier L, Collilieux X (2016) ITRF2014: A new release of the International Terrestrial Reference Frame modeling nonlinear station motions. J Geophys Res 121:6109-6131. https://doi.org/10. 1002/2016JB013098

Anderson JM, Xu MH (2018) Source structure and measurement noise are as important as all other residual sources in geodetic VLBI combined. Journal of Geophysical Research: Solid Earth 123(11):10,162-10,190. https:// doi.org/10.1029/2018JB015550

Artz T, Halsig S, Iddink A, Nothnagel A (2016) ivg::ASCOT: Development of a New VLBI Software Package. In: Behrend D, Baver KD, Armstrong KL (eds) IVS 2016 General Meeting Proceedings "New Horizons with VGOS", NASA/ CP-2016-219016, pp 217-221, https://doi.org/10.22323/1.344.0140, https://ivscc.gsfc.nasa.gov/publications/gm2016/045 artz_etal.pdf

Bizouard C, Lambert S, Gattano C, Becker O, Richard JY (2019) The IERS EOP 14C04 solution for Earth orientation parameters consistent with ITRF 2014. J Geodesy 93(5):621-633. https://doi.org/10.1007/ s00190-018-1186-3

BKG (2020) VTRF2020b combined solution. https://ccivs.bkg.bund.de/combi nation/QUAT/SNX/NTRF2020b_IVS.snx, Accessed: 2021-02-26

Bolotin S, Baver KD, Gipson J, Gordon D, MacMillan D (2012) The first release of vsolve. In: Behrend D, Baver KD (eds) IVS 2012 General Meeting Proceedings "Launching the Next-Generation IVS Network", NASA/CP-2012217504, pp 222-226, http://ivscc.gsfc.nasa.gov/publications/gm2012/ bolotin.pdf

Bolotin S, Baver KD, Gipson J, Gordon D, MacMillan D (2015) Implementation of the vgosdb format. In: Haas R, Colomer F (eds) Proceedings of the 22nd European VLBI Group for Geodesy and Astrometry Working Meeting, pp 150-152, http://www.oso.chalmers.se/evga/22_EVGA_2015_ Ponta_Delgada.pdf

Bradley BK, Sibois A, Axelrad P (2016) Influence of ITRS/GCRS implementation for astrodynamics: coordinate transformations. Advances in Space Research 57(3):850-866. https://doi.org/10.1016/j.asr.2015.11.006

Charlot P, Jacobs CS, Gordon D, Lambert S, de Witt A, Böhm J, Fey AL, Heinkelmann R, Skurikhina E, Titov O, Arias EF, Bolotin S, Bourda G, Ma C, Malkin Z, Nothnagel A, Mayer D, MacMillan DS, Nilsson T, Gaume R (2020) The third realization of the international celestial reference frame by very long baseline interferometry. Astron Astrophys 644:A159. https://doi.org/10. 1051/0004-6361/202038368

Corbin A, Haas R (2019) Scheduling of Twin Telescopes and the Impact on Troposphere and UT1 Estimation. In: Haas R, Garcia-Espada S, López Fernández JA (eds) Proceedings of the 24th European VLBI for Geodesy and Astrometry (EVGA) working meeting, ISBN: 978-84-4165634-5, pp 194-98

Deller AT, Brisken WF, Phillips CJ, Morgan J, Alef W, Cappallo R, Middelberg E, Romney J, Rottmann H, Tingay SJ, Wayth R (2011) DiFX-2: A More Flexible, 
Efficient, Robust, and Powerful Software Correlator. Publications of the Astronomical Society of the Pacific 123(901):275. https://doi.org/10.1086/ 658907

Gipson J, Baver KD (2015) Minimization of the UT1 Formal Error Through a Minimization Algorithm. In: Haas R, Colomer F (eds) Proceedings of the 22th European VLBI for Geodesy and Astrometry (EVGA) working meeting, ISBN: 978-989-20-6191-7, pp 230-234

Haas R, Casey S, Conway J, Elgered G, Hammargren R, Helldner L, Johansson KÅ, Kylenfall U, Lerner M, Pettersson L, Wennerbäck L (2019) Status of the Onsala Twin Telescopes - Two Years After the Inauguration. In: Haas R, Garcia-Espada S, López Fernández JA (eds) Proceedings of the 24th European VLBI for Geodesy and Astrometry (EVGA) working meeting, ISBN: 978-84-416-5634-5, pp 5-9

IERS (2021) International Earth Rotation and Reference Systems Service. https://iers.org, Accessed 26 Feb 2021

Leek J, Artz T, Nothnagel A (2015) Optimized scheduling of VLBI UT1 intensive sessions for twin telescopes employing impact factor analysis. Journal of Geodesy 89:911-924. https://doi.org/10.1007/s00190-015-0823-3

Luzum B, Gambis D (2014) Explanatory Supplement to IERS Bulleting A and Bulletin B/C04.ftp://hpiers.obspm.fr/iers/bul/bulb_new/bulletinb.pdf

MIT/Haystack (2019) Vgos data processing manual v1.1. https://www.haystack. mit.edu/wp-content/uploads/2020/07/docs_hops_000_vgos-data-proce ssing.pdf, Accessed 24 Apr 2020

MIT/Haystack (2020) Haystack Observatory Postprocessing System (HOPS). https://www.haystack.mit.edu/haystack-observatory-postprocessingsystem-hops/, Accessed 24 Apr 2020

Niell A, Whitney A, Petrachenko B, Schlüter W, Vandenberg N, Hase H, Koyama Y, Ma C, Schuh H, Tuccari G (2006) VLBI2010: Current and future requirements for geodetic VLBI systems (WG3 - final report)

Niell A, Barrett J, Burns A, Cappallo R, Corey B, Derome M, Eckert C, Elosegui P, McWhirter R, Poirier M, Rajagopalan G, Rogers AEE, Ruszczyk C, SooHoo J, Titus M, Whitney A, Behrend D, Bolotin S, Gipson J, Gordon D, Himwich E, Petrachenko B (2018) Demonstration of a Broadband Very Long Baseline Interferometer System: A New Instrument for High-Precision Space Geodesy. Radio Science 53. https://doi.org/10.1029/2018RS006617

Nilsson T, Haas R (2010) Impact of atmospheric turbulence on geodetic very long baseline interferometry. Journal of Geophysical Research: Solid Earth 115(B3), https://doi.org/10.1029/2009JB006579
Nothnagel A, Campbell J (1991) Polar motion observed by daily vlbi measurements. In: Proceedings of the AGU chapman conference on geodetic VLBI: monitoring global change, NOAA Technical Report NOS 137 NGS 49, p 345-354

Nothnagel A, Artz T, Behrend D, Malkin Z (2017) International VLBI Service for Geodesy and Astrometry - Delivering high-quality products and embarking on observations of the next generation. Journal of Geodesy 91(7):711-721. https://doi.org/10.1007/s00190-016-0950-5

Petrachenko B, Niell A, Behrend D, Corey B, Böhm J, Charlot P, Collioud A, Gipson J, Haas R, Hobiger T, Koyama Y, MacMillan D, Malkin Z, Nilsson T, Pany A, Tuccari G, Whitney A, Wresnik J (2009) Design aspects of the VLBI2010 system. Progress report of the IVS VLBI2010 committee

Plag HP, Pearlman M (2009) Global Geodetic Observing System. Springer. https://doi.org/10.1007/978-3-642-02687-4

Schartner M, Böhm J (2019) VieSched++: A new VLBI scheduling software for geodesy and astrometry. Publications of the Astronomical Society of the Pacific 131(1002):084501. https://doi.org/10.1088/1538-3873/ab1820

Sovers OJ, Fanselow JL, Jacobs CS (1998) Astrometry and geodesy with radio interferometry: experiments, models, results. Reviews of Modern Physics 70(4):1393-1454. https://doi.org/10.1103/RevModPhys.70.1393

Uunila M, Nothnagel A, Leek J (2012) Influence of Source Constellations on UT1 Derived from IVS INT1 Sessions. In: Behrend D, Baver KD (eds) Proceedings of the 7th General Meeting (GM2012) of the International VLBI Service for Geodesy and Astrometry (IVS), NASA/CP-2012-217504, pp 395-399

Varenius E, Haas R (2020) Short-baseline interferometry co-location experiments at the Onsala Space Observatory. arXiv e-prints arXiv:2010.16214,

Wakasugi T, Kurihara S, Ueshiba H, Umei M, Ishigaki M, Munekane H (2019) Current Status of VGOS Observation with Ishioka VLBI Station. In: Armstrong KL, Baver KD, Behrend D (eds) IVS 2018 General Meeting Proceedings, "Global Geodesy and the Role of VGOS - Fundamental to Sustainable Development", NASA/CP-2019-219039, pp 61-65

\section{Publisher's Note}

Springer Nature remains neutral with regard to jurisdictional claims in published maps and institutional affiliations.

\section{Submit your manuscript to a SpringerOpen ${ }^{\odot}$ journal and benefit from:}

- Convenient online submission

- Rigorous peer review

- Open access: articles freely available online

- High visibility within the field

- Retaining the copyright to your article

Submit your next manuscript at $\boldsymbol{\nabla}$ springeropen.com 